\title{
Bounded-Collusion Identity-Based \\ Encryption from Semantically-Secure Public-Key Encryption: \\ Generic Constructions with Short Ciphertexts
}

\author{
Stefano Tessaro ${ }^{1, \star}$ and David A. Wilson ${ }^{2}$ \\ 1 University of California, Santa Barbara \\ 2 MIT
}

\begin{abstract}
To circumvent the lack of generic constructions of identitybased encryption (IBE), Dodis et al. (EUROCRYPT '02) introduced the notion of bounded-collusion IBE (BC-IBE), where attackers only learn secret keys of an a-priori bounded number $t$ of identities. They provided a generic BC-IBE construction from any semantically-secure encryption scheme which, however, suffers from a $\omega(t)$ blow-up in ciphertext size. Goldwasser et al. (TCC 2012) recently presented a generic construction with no ciphertext-length blow-up. Their construction requires an underlying public-key scheme with a key homomorphism, as well as a hash-proof-style security definition that is strictly stronger than semantic security. This latter requirement in particular reduces the applicability of their construction to existing schemes.

In this paper, we present the first generic constructions of BC-IBE from semantically-secure encryption schemes with no ciphertext-length blowup. Our constructions require different degrees of key-homomorphism and malleability properties that are usually easy to verify. We provide concrete instantiations based on the DDH, QR, NTRU, and LWE assumptions. For all of these assumptions, our schemes present the smallest BC-IBE ciphertext size known to date. Our NTRU-based construction is particularly interesting, due to the lack of NTRU-based IBE constructions as well as the fact that it supports fully-homomorphic evaluation.

Our results also yield new constructions of bounded CCA-secure cryptosystems.
\end{abstract}

\section{Introduction}

PUBLIC-KEY ENCRYPTION. One of the classic and best-studied models of secure communication is that of public-key encryption (PKE) [12], in which each individual independently generates a public-key / secret-key pair. Anyone possessing the public key can encrypt a message such that only the individual with the associated secret key can decrypt. To date, there are innumerable PKE constructions proven secure based on a wide variety of hardness assumptions.

\footnotetext{
^ Research done while the author was with MIT CSAIL.
} 
However, the basic public-key model lacks a well-developed structure for public key verification. One can encrypt messages using a public key, but the model implies a trust that the public key belongs to a specific individual, unless an expensive public-key infrastructure is in place. In order to make explicit these assumptions and avoid potential difficulties with key distribution, cryptographers have explored other models of encryption.

IDENTITY-BASED ENCRYPTION. The identity-based encryption (IBE) model, introduced by Shamir in 1984 [30, attempts to alleviate the above concerns. In this model, a trusted center generates a master secret key and public parameters for the entire system. Anyone can encrypt a message to any user of the system using only these global public parameters and the user's identity. To decrypt, a user must obtain the secret key for their identity from the trusted center (who presumably authenticates the user before distributing the key).

The security model for IBE assumes that the adversary can adaptively obtain an arbitrary number of secret keys for users in the system, and requires that messages encrypted to any other user still be indistinguishable to the adversary. This models the idea that an individual's messages are still secure even if an arbitrary number of other users of the system collude against that user.

The first constructions of IBE came in 2001, by Boneh and Franklin [5] and Cocks 9. Both of these constructions assumed the existence of random oracles; however, subsequent work by Boneh and Boyen [3] and Waters 32] achieved IBE in the standard model. There now exist a number of IBE constructions in both the random oracle and standard models, under hardness assumptions of problems in bilinear groups (e.g. [5/7,3/32]), various forms of the Quadric Residuosity (QR) problem (e.g. 96]), and the Learning With Errors problem (e.g. 2081]). Some of these, and in particular all those based on the standard QR problem, additionally require random oracles. However, no constructions of IBE are known from generic primitives.

BoundED-COLLUSION IBEs. As an attempt to come up with constructions under a wider range of assumptions, cryptographers began looking at a variant of IBE known as Bounded-Collusion IBE (BC-IBE). In this model, one only guarantees security against an adversary who obtains secret keys associated with at most $t$ identities, where the size of the parameters of the system are allowed to depend on $t$. Falling short of achieving full security, the bounded-collusion model can be a realistic assumption in many settings, and is in fact a necessary restriction to achieve the more general notion of functional encryption [24]. Additionally, it has been studied in other settings, notably broadcast encryption and revocation (e.g. 171819 27/29 2513]).

The first construction of BC-IBE came in the context of key-insulated systems in [15]. This paper gave a general reduction from any semantically secure publickey cryptosystem to a BC-IBE scheme. However, their construction suffers from a large ciphertext-size blowup - the resulting ciphertext length is a factor $\omega(t)$ larger than that of the underlying encryption scheme. To mitigate this, this work was recently followed by that of Goldwasser et al. 22]: They provide a new construction that relies on a public-key encryption scheme which exhibits 
key-homomorphic properties, i.e., secret keys and public keys are elements of respective groups (with possibly different operations, which we denote by + and $\cdot)$, and there exists a homomorphism $\mu$ such that $\mu\left(\mathrm{sk}+\mathrm{sk}^{\prime}\right)=\mu(\mathrm{sk})+\mu\left(\mathrm{sk}^{\prime}\right)$, where $\mu(\mathbf{s k})$ and $\mu\left(\mathbf{s k}^{\prime}\right)$ are valid public keys for which sk and sk' yield correct decryption, respectively. More concretely, the GLW construction generates multiple public-key / secret-key pairs $\left(\mathrm{pk}_{1}, \mathrm{sk}_{1}\right), \ldots,\left(\mathrm{pk}_{n}, \mathrm{sk}_{n}\right)$, letting the public parameters and the master secret key of the scheme be $\mathrm{pp}=\left(\mathrm{pk}_{1}, \ldots \mathrm{pk}_{n}\right)$ and $\mathrm{msk}=\left(\mathrm{sk}_{1}, \ldots, \mathrm{sk}_{n}\right)$, respectively. Then, an efficient map $\phi$ associates every identity ID with a vector $\left[\mathrm{id}_{1}, \ldots, \mathrm{id}_{n}\right]$, and a message $m$ is encrypted for an identity ID as the ciphertext $c=\operatorname{Enc}\left(\mathrm{pk}_{\mathrm{ID}}, m\right)$, where $\mathrm{pk}_{\mathrm{ID}}=\prod_{i=1}^{n} \mathrm{pk}_{i}^{\mathrm{id}_{i}}$. By the existence of $\mu$, this ciphertext can be decrypted using sk $\mathrm{ID}_{\mathrm{ID}}=\sum_{i=1}^{n} \mathrm{id}_{i} \cdot \mathrm{sk}_{i}$, since the homomorphism guarantees that $\mathrm{pk}_{\mathrm{ID}}=\mu\left(\mathrm{sk}_{\mathrm{ID}}\right)$. The map $\phi$ is subjected to a combinatorial requirement that disallows computing $s k_{I D}$ given $s_{I_{D}}$ for $t$ different ID $^{\prime} \neq$ ID. The GLW construction preserves the ciphertext size of the underlying encryption, but its security requires the latter to satisfy a property which is strictly stronger than semantic security. This property is inspired by the security of hash-proof systems [11, and in particular does not allow the homomorphism $\mu$ to be one-to-one. This somewhat hinders the applicability of their framework to existing encryptions schemes not designed with this security goal in mind.

OUR CONTRIBUTIONS. In this paper, we seek for generic constructions of BCIBE which rely on encryption schemes that solely satisfy the standard security notion of semantic security in addition to some syntactical, non-security-related, properties which can be easily verified. Our constructions have the added benefit of conceptual simplicity, and the resulting instantiations from concrete assumptions either outperform or abstract existing BC-IBE constructions along different axes.

In summary, this paper makes three main contributions:

1. As our first contribution, we revisit the GLW approach in the context of selective security. The latter security notion only demands security for attackers attempting to break the confidentiality of messages encrypted for an a-priori specified identity (in particular, independently of the parameters of the scheme). We prove that the GLW approach is selectively secure for every semantically secure encryption scheme with key-homomorphic properties whenever $\phi$ satisfies a slightly stronger property that the one used in 22, namely that of cover-freeness introduced in [16] and used in several other works (e.g. 27 1014], and others). While being strictly weaker than the notion of full security, selective security is sufficient for some applications, as discussed below.

2. Whenever the underlying semantically-secure scheme satisfies an additional new property - which we call weak multi-key malleability - we prove that the GLW construction achieves full BC-IBE security, i.e., confidentiality holds even with respect an identity chosen adaptively after learning the parameters of the schemes as well as secret keys for at most $t$ other identities. 
Roughly, our malleability property states that given the encryption of $c=$ Enc $(\mathrm{pk}, m)$ of an unknown message $m$ under a known public key pk, and given an additional public-key / secret-key pair $\left(\mathrm{pk}^{\prime}, \mathrm{sk}^{\prime}\right)$, we can efficiently produce a ciphertext which is indistinguishable from an encryption of $m$ under pk $\cdot \mathrm{pk}^{\prime}$. An example scheme with this property is ElGamal encryption - hence we directly obtain a DDH-based BC-IBE scheme from ElGamal encryption.

3. As our third contribution, we provide a new, alternative construction that relies on a different form of malleability (which we simply call multi-key malleability), and does not require any explicit key-homomorphic structure. Intuitively, our notion requires that given $c=\operatorname{Enc}(\mathrm{pk}, m)$ for an unknown message $m$, and another public key $\mathrm{pk}^{\prime}$, we can obtain a new ciphertext $c$ which decrypts to $m$ under a combination of the secret keys sk and sk' associated with pk and $\mathrm{pk}^{\prime}$. We provide an efficient instantiation based on NTRU [26], exploiting it multi-key homomorphic properties recently observed by Lopez-Alt et al. [28. This is of particular interest due to the fact that no fully-secure NTRU-based IBE scheme is known to date. Moreover, our constructions support homomorphic evaluation of ciphertexts, and this is the only construction of identity-based fully homomorphic encryption beyond the recent result by Gentry, Sahai, and Waters [21].

To conclude, we stress that our instantiation of the GLW approach is somewhat orthogonal to the one by Goldwasser et al:: Our instantiation requires indeed somewhat larger public-parameters at the cost of a weaker assumption on the underlying encryption scheme, hence leading to wider applicability and often smaller ciphertexts. Nonetheless, we believe that large ciphertexts are generally a more limiting factor than large parameters, especially in settings where many messages are encrypted with the same parameters.

A summary of our instantiations and their parameters is given in Table 1 comparing them to previously known best constructions. For LWE and NTRU, the best previously known construction was obtained by using the construction of [15. We also provide a construction based on QR which does not outperform the one of [22], even though we find it conceptually simpler.

FROM IBE TO CCA-SECURITY. A somewhat related problem is that of building bounded-CCA secure public-key encryption [10]: Concretely, for $t$-bounded CCA security, semantic security must hold also for attackers which can decrypt up to $t$ ciphertexts other than the challenge ciphertext for which we attempt to break confidentiality. We note that by re-interpreting a result of Boneh et al. [4, every construction of a BC-IBE scheme selectively secure against $t$-collusions directly yields a $t$-bounded CCA secure PKE. Hence, our BC-IBE constructions also directly yield better bounded-CCA-secure constructions, in terms of ciphertext size and/or conceptual simplicity. When applying our framework to ElGamal, for example, we obtain a construction which is equivalent to the one proposed in 10, for which a direct security proof was given. Moreover, our instantiation from NTRU is indeed more efficient than the best fully CCA-secure construction from NTRU given by Steinfeld et al. 31. 
Table 1. Comparison with previous works on BC-IBE. Here $t$ is the collusion parameter and $|\mathcal{I D}|$ is the total number of identities in the system. PK and ciphertext size implicitly include the security parameter. The upper section of the table considers generic constructions, whereas the lower section describes existing constructions from concrete assumptions. Note that linear hash proof property implies semantic security, while being strictly stronger than it.

\begin{tabular}{|c|l|l|l|}
\hline Construction & \multicolumn{1}{|c|}{ Assumptions } & Ciphertext size & \multicolumn{1}{|c|}{ PK size } \\
\hline \hline DKXY02 [15] & $\begin{array}{l}\text { Semantically secure } \\
\text { PKE }\end{array}$ & $\begin{array}{l}\Theta(t \log |\mathcal{I} \mathcal{D}|) \\
\text { PKE ciphertexts }\end{array}$ & $\Theta\left(t^{2} \log |\mathcal{I D}|\right)$ PKE PKs \\
\hline GLW12 22] & $\begin{array}{l}\text { PKE w/linear hash } \\
\text { proof and key homo- } \\
\text { morphism }\end{array}$ & $\begin{array}{l}\text { Same as } \\
\text { underlying PKE }\end{array}$ & $\Theta(t \log |\mathcal{I D}|)$ PKE PKs \\
\hline This work & $\begin{array}{l}\text { Semantic-secure PKE; } \\
\text { key homomorphism, } \\
\text { weak multi-key mal- } \\
\text { leability }\end{array}$ & $\begin{array}{l}\text { Same as } \\
\text { underlying PKE }\end{array}$ & $\Theta\left(t^{2} \log |\mathcal{I D}|\right)$ PKE PKs \\
\hline This work & $\begin{array}{l}\text { Semantic-secure PKE; } \\
\text { multi-key malleability }\end{array}$ & $\begin{array}{l}\text { Same as } \\
\text { underlying PKE }\end{array}$ & $\Theta\left(t^{2} \log |\mathcal{I D}|\right)$ PKE PKs \\
\hline \hline DKXY02 [15] & DDH & 3 group elements & $\Theta(t)$ group elements \\
\hline GLW12[22] & DDH & 3 group elements & $\Theta(t \log |\mathcal{I D}|)$ group elts \\
\hline This work & DDH & 2 group elements & $\Theta\left(t^{2} \log |\mathcal{I D}|\right)$ group elts \\
\hline GLW12 22] & QR & $\begin{array}{l}2 \text { RSA group } \\
\text { elements }\end{array}$ & $\Theta(t \log |\mathcal{I D}|)$ group elts \\
\hline This work & LWE & $\begin{array}{l}\text { Same as } \\
\text { GPV 20 }\end{array}$ & $\Theta\left(t^{2} \log |\mathcal{I D}|\right)$ GPV PKs \\
\hline This work & NTRU & $\begin{array}{l}\text { Same as NTRU- } \\
\text { Encrypt 26 }\end{array}$ & $\Theta\left(t^{2} \log |\mathcal{I D}|\right)$ NTRU PKs \\
\hline
\end{tabular}

\section{Preliminaries}

\subsection{Public-Key Encryption}

PKE SyntaX. As usual, a public-key encryption (PKE) scheme is a triple of efficient algorithms PKE = (Gen, Enc, Dec) where:

- Gen is the (randomized) key generation algorithm: it takes no input (other than the security parameter $1^{k}$, which is implicit and generally omitted), and outputs a public-key / secret-key pair (pk, sk).

- Enc and Dec are the (randomized) encryption and the (deterministic) decryption algorithms, such that for all valid public-key / secret-key pairs (pk, sk) output by Gen, and all messages $m$, the probability $\mathrm{P}[\operatorname{Dec}(s k, \operatorname{Enc}(\mathrm{pk}, m)) \neq$ $m$ ] is negligible, where the probability is taken over the random coins of the encryption algorithm Enc.

Often, we allow public-key encryption schemes to additionally depend on explicit public parameters pp (randomly generated in an initial phase and shared across multiple instances of the PKE scheme) on which all of Gen, Enc, and Dec 
are allowed to depend. Examples include the description of a group $G$ with its generator $g$. We will often omit them in the descriptions of generic constructions from PKE schemes.

SECURITY OF PKE. We define security against chosen-plaintext attacks (for short, IND-CPA security) 232 for a PKE scheme PKE = (Gen, Enc, Dec) via a security game involving an adversary $\mathcal{A}$ which is initially given the public key pk, and subsequently outputs a pair of equal-length messages $m_{0}, m_{1}$. The adversary continues after receiving a challenge ciphertext $c^{*} \stackrel{\$}{\leftarrow} \mathrm{Enc}\left(\mathrm{pk}, m_{b}\right)$ for a random secret bit $b$, and then finally outputs a guess $b^{\prime}$ for $b$. We say that $\operatorname{PKE}$ is $(\tau, \varepsilon)$ ind-cpa-secure if all attackers $\mathcal{A}$ with time complexity at most $\tau$ guess the right bit (i.e., $b^{\prime}=b$ ) with probability at most $\frac{1+\varepsilon}{2}$. Moreover, it is simply ind-cpa secure if for all polynomials $p$, there exists a negligible function $\nu$ such that the scheme is $(p(k), \nu(k))$-ind-cpa-secure for all values of the security parameter $k$. We also consider security against chosen ciphertext attacks (for short, IND-CCA security), where the adversary is additionally able to decrypt ciphertexts under the constraint that a decryption query for the challenge ciphertext is never asked. We say that PKE is $(\tau, t, \varepsilon)$-ind-cca-secure if any attacker with time complexity $\tau$ and making at most $t$ decryption queries guesses $b$ with probability at most $\frac{1+\varepsilon}{2}$. The asymptotic notion of $t$-ind-cca-secure is defined accordingly.

\subsection{Identity-Based Encryption}

Recall that an identity-based encryption (IBE) scheme for identity set $\mathcal{I D}$ is a 4-tuple of algorithms IBE = (IBEGen, IBEExtract, IBEEnc, IBEDec) satisfying the following syntactical properties:

- IBEGen is the randomized parameter generator algorithm which returns a pair (msk, pp), where msk is the so-called master secret key, and pp are the public parameters.

- The extraction algorithm IBEExtract, on input the master secret-key msk and a valid identity ID $\in \mathcal{I D}$ returns a secret key sk ID $\stackrel{\$}{\leftarrow}$ IBEExtract(msk, ID) associated with this identity.

- The encryption algorithm IBEEnc takes as inputs the public parameters $\mathrm{pp}$, an identity ID $\in \mathcal{I D}$, and a message $m$, and returns a ciphertext $c \stackrel{\$}{\leftarrow}$ IBEEnc(pp, ID,$m)$ such that for the associated deterministic algorithm IBEDec, IBEDec(skID, IBEEnc $(p p$, ID,$m))=m$ with overwhelming probability for each (pp, msk) output by Gen and $s k_{\text {ID }}$ output by IBEExtract(msk, ID).

The notion of IND-CPA security is extended to the setting of IBE. The adversary, given the public parameters pp, can obtain keys sk $\mathrm{ID}_{\mathrm{D}}$ for identities ID of its choice (via so-called extraction queries), and outputs at some point a pair of equal-length challenge messages $m_{0}, m_{1}$, together with a challenge identity ID* for which no extraction query has been issued. It then obtains an encryption of $m_{b}$ for the challenge identity ID* and for a random bit $b$. The adversary is asked to guess $b$, constrained on not asking a key extraction query for ID* . We also consider a weaker security notion, called selective IND-CPA security: 
Here, the adversary is required to choose its challenge identity beforehand, and only subsequently learns the public parameters and is given access to the IBEExtract oracle.

In analogy to the case of conventional PKE, we say that IBE is $(\tau, t, \varepsilon)$-cpasecure if all $\tau$-time adversaries $\mathcal{A}$ making $t$ extraction queries output $b$ with probability at most $\frac{1+\varepsilon}{2}$ in the CPA-security game above. Similarly, we define $(\tau, t, \varepsilon)$-selective-cpa-secure likewise for the selective-CPA game above, as well as the asymptotic notions of $t$-cpa and $t$-selective-cpa security.

\section{Revisiting the GLW Construction}

In the first part of this paper, we revisit the IBE construction for boundedcollusion security proposed by Goldwasser, Lewko, and Wilson 22] - henceforth, we refer to this construction as the GLW construction. We show two generic results, the first one for selective security and the second one for full IBE security. Then, we discuss a new instantiation of this paradigm based on DDH. Two more instantiations based on the LWE and QR assumptions are deferred to the full version for lack of space.

\subsection{The GLW Construction}

Secret-key to Public-key Homomorphisms. Throughout this section, we (tacitly) consider only public-key cryptosystems PKE $=$ (Gen, Enc, Dec) with the property that secret and public keys are elements of groups $G$ and $H$, respectively. For convenience and ease of distinction, we will denote the group operations on $G$ and $H$ as + and ', respectively.

Definition 1 (Secret-key to public-key homomorphism). We say that PKE admits a secret-key to public-key homomorphism if there exists a map $\mu: G \rightarrow H$ such that:

(i) $\mu$ is a homomorphism, i.e., for all sk, $\mathrm{sk}^{\prime} \in G$, we have $\mu\left(\mathrm{sk}+\mathrm{sk}^{\prime}\right)=$ $\mu(\mathrm{sk}) \cdot \mu\left(\mathrm{sk}^{\prime}\right)$;

(ii) Every output (sk, pk) of Gen satisfies $\mathrm{pk}=\mu(\mathrm{sk})$.

We stress that we are not requiring that every element sk $\in G$ is a valid secret key output by Gen. This will be important in our LWE instantiation below. In this case, we still want to make sure that decryption is correct: In particular, we say below that $\mu$ satisfies $n$-correctness if for any $n^{\prime} \leq n$ valid secret keys $\mathrm{sk}_{1}, \ldots, \mathrm{sk}_{n^{\prime}}$ output by Gen, the probability $\mathrm{P}[\operatorname{Dec}(\mathrm{sk}, \operatorname{Enc}(\mu(\mathrm{sk}), m)) \neq m]$ is negligible for all messages $m$, where the probability is over the coins of Enc and where $\mathrm{sk}=\mathrm{sk}_{1}+\cdots+\mathrm{sk}_{n^{\prime}}$. (This property is implicitly satisfied for all $n$ if all elements of $G$ are valid secret keys.)

Also note that the map $\mu$ does not need to be efficiently computable for our applications, even though the map is often very efficient. Additionally, we observe that in case the scheme depends on some explicit public parameter (like a generator or a matrix, as will be the case in our examples below), $\mu$ is indeed allowed to be parameter-dependent. 
The GLW Construction. Goldwasser, Lewko, and Wilson 22 presented a generic approach to build a bounded-collusion secure IBE from a public-key encryption scheme admitting a secret-key to public-key homomorphism. Specifically, let PKE = (Gen, Enc, Dec) be such a public-key encryption scheme with homomorphism $\mu: G \rightarrow H$ satisfying $n$-correctness, and let $\phi: \mathcal{I D} \rightarrow\{0,1\}^{n}$ be a polynomial-time computable function, called the identity map. (With a slight abuse of notation, it will be convenient to consider the output $\phi$ as a subset of $\{1, \ldots, n\}$, encoded in the canonical way as an $n$-bit string.) Then, the GLW construction for PKE and $\phi$ gives rise to the following IBE scheme IBE $=($ IBEGen, IBEExtract, IBEEnc, IBEDec) with identities from the set $\mathcal{I D}$ defined as follows (where additionally IBEDec $\left(\operatorname{sk}_{\mathrm{ID}}, c\right)=\operatorname{Dec}\left(\mathrm{sk}_{\mathrm{ID}}, c\right)$ )

\begin{tabular}{|c|c|c|}
\hline IBEGen: & IBEExtract(sk, ID): & IBEEnc(pk, ID, $m)$ : \\
\hline $\begin{array}{l}(\mathbf{p k}, \mathbf{s k}) \stackrel{\$}{\leftarrow} \mathrm{Gen}^{n} \\
\mathrm{msk} \leftarrow \mathbf{s k} \\
\mathrm{pp} \leftarrow \mathbf{p k} \\
\operatorname{Return}(\mathrm{msk}, \mathrm{pp})\end{array}$ & $\begin{array}{l}\operatorname{sk}_{\mathrm{ID}}=\sum_{i \in \phi(\mathrm{ID})} \mathrm{sk} \\
\text { Return sk }[i]\end{array}$ & $\begin{array}{l}\mathrm{pk}_{\mathrm{ID}}=\prod_{i \in \phi(\mathrm{ID})} \mathbf{p k}[i] \\
c \stackrel{\$}{\leftarrow} \operatorname{Enc}\left(\mathrm{pk}_{\mathrm{ID}}, m\right) \\
\operatorname{Return~} c\end{array}$ \\
\hline
\end{tabular}

The notation (pk, sk) $\stackrel{\$}{\leftarrow}$ Gen $^{n}$ denotes running Gen $n$ times, with independent random coins, and $\mathbf{p k}, \mathbf{s k}$ are vectors such that $(\mathbf{p k}[i], \mathbf{s k}[i])$ is the output of the $i$-th execution of Gen. First note that correctness of IBE follows trivially from the correctness of PKE and the existence of a secret-key to public-key homomorphism $\mu$ with $n$-correctness, since $\mathrm{pk}_{\mathrm{ID}}=\mu\left(\mathrm{sk}_{\mathrm{ID}}\right)$ holds for all IDs and sk $\mathrm{ID}_{\mathrm{ID}}$ is the sum of at most $n$ valid secret keys. We stress that a central advantage of the above construction is that IBE ciphertexts are ciphertexts of the underlying encryption scheme PKE. Also, note that if PKE relies on some public parameters, these are generated once and used across all uses of Gen, Enc, and Dec.

InSTANTIATING THE IDENTITY MAP. We still need to discuss how the map $\phi$ is instantiated. In all constructions of this paper, we rely on constructions based on cover-free sets, following previous work on bounded-collusion IBE [15, boundedCCA security [10, and bounded security for FDH signatures [14]. Concretely, let $2^{[n]}$ be the set of subsets of $[n]:=\{1, \ldots, n\}$.

Definition 2 (Cover-free sets). We say that $\phi: \mathcal{I D} \rightarrow 2^{[n]}$ is $(t, s)$-cover free if $|\phi(x)|=s$ for all $x \in \mathcal{I D}$, and moreover $\phi\left(x_{t}\right) \backslash \bigcup_{i=1}^{t-1} \phi\left(x_{i}\right) \neq \emptyset$ for all $x_{1}, \ldots, x_{t} \in$ $\mathcal{I D}$, i.e., the set $\phi\left(x_{t}\right)$ is not covered by the union of $\phi\left(x_{1}\right), \ldots, \phi\left(x_{t-1}\right)$.

In general, we will equivalently think of $\phi$ as a map $\mathcal{I D} \rightarrow\{0,1\}^{n}$, where we output the characteristic vector of the associated set, instead of the set itself. The following gives the currently best-known construction of cover-free sets.

Theorem 1 ([10]). For all integers $t \geq 1$, there exists a polynomial-time computable $(t, s)$-cover-free map $\phi: \mathcal{I D} \rightarrow\{0,1\}^{n}$, where $n=16 t^{2} \log |\mathcal{I D}|$ and $s=4 t \log |\mathcal{I D}|$. 
We note that Goldwasser, Lewko, and Wilson used a weaker requirement of $\phi$ that only requires linear independence of the vectors $\phi\left(x_{1}\right), \ldots, \phi\left(x_{t}\right)$. In this case, the output length $n$ can be reduced to $O(t \log |\mathcal{I D}|)$, or even $O(t)$ if we allow both identities as well as components of $\phi(x)$ to be elements of $\mathbb{Z}_{p}$ for some large prime $p$. However, the price they pay compared to our results below is that the underlying encryption scheme is required to satisfy a harder to show notion than in our results given below assuming cover-freeness, and this is often reflected in instantiations with larger ciphertexts.

\subsection{Selective Security of the GLW Construction}

We start with selective security, which will be important to obtain bounded CCA-secure cryptosystems with short ciphertexts, as we explain below in Section 5. In the following, let PKE = (Gen, Enc, Dec) be an arbitrary public-key encryption scheme which admits secret-key to public-key homomorphism, and let IBE be the IBE scheme resulting from the above construction, using an underlying identity map $\phi$.

Theorem 2 (Selective ID Security of GLW). Assume that PKE is indcpa-secure, and that $\phi$ is $(t+1, s)$-cover free. Then, the GLW construction is t-selective-cpa-secure.

Proof. Let $\mathcal{A}$ be a selective-cpa adversary for IBE which outputs $b^{\prime}=b$ with probability at least $(1+n \varepsilon) / 2$, and which makes at most $t$ extraction queries. We construct an ind-cpa adversary $\mathcal{B}$ for PKE from $\mathcal{A}$, guessing the bit $b$ with probability $\frac{1+\varepsilon}{2}$. Concretely, the adversary $\mathcal{B}$ first runs $\mathcal{A}$, obtaining the challenge identity ID*, and chooses an index $i^{*}$ uniformly at random from the set $S^{*}=$ $\left\{i: \mathrm{id}_{i}^{*}=1\right\}$, where $\phi\left(\mathrm{ID}^{*}\right)=\left[\mathrm{id}_{1}^{*}, \ldots, \mathrm{id}_{n}^{*}\right]$. It then gets a public key $\mathrm{pk}^{*}$ from the underlying CPA game, and computes $(\mathbf{p k}[j], \mathbf{s k}[j]) \stackrel{\$}{\leftarrow}$ Gen for all $j \in[n] \backslash\left\{i^{*}\right\}$. Finally, it sets $\mathbf{p k}\left[i^{*}\right]=\mathbf{p k}^{*} \cdot\left(\prod_{j \neq i^{*}} \mathbf{p k}[j]^{-\mathrm{id}_{j}^{*}}\right)$.

The adversary $\mathcal{B}$ then gives $\mathrm{pp}=$ pk to $\mathcal{A}$ and runs it until it outputs a pair $\left(m_{0}, m_{1}\right)$. In particular, $\mathcal{A}$ 's extraction queries for ID $\neq$ ID $^{*} \in \mathcal{I D}$ are replied by computing $\left[\mathrm{id}_{1}, \ldots, \mathrm{id}_{n}\right]=\phi(\mathrm{ID})$ and, if $\mathrm{id}_{i^{*}}=0$, returning $\mathrm{sk}_{I D}:=\sum_{i} \mathrm{id}_{i} \cdot \mathbf{s k}[i]$. Note that if $\mathrm{id}_{i^{*}}=1$, then $\mathcal{B}$ cannot answer the extraction query, as it does not know any corresponding $\mathbf{s k}\left[i^{*}\right]$. In this case, it returns $\perp$, and sets a flag bad to true. When the adversary $\mathcal{A}$ outputs a pair $\left(m_{0}, m_{1}\right)$ of messages of equal length, $\mathcal{B}$ forwards them to the CPA, obtaining a challenge ciphertext $c^{*}$, which it then gives back to $\mathcal{A}$, and its simulated execution is continued until it outputs a bit $b^{\prime}$. To conclude, $\mathcal{B}$ outputs the bit $b^{\prime}$ if bad is not set to true, and returns a random bit otherwise. Note that we have $\mathrm{pk}_{\mathrm{ID}^{*}}=\mathrm{pk}^{*}$ by our definition.

Since $\phi$ is $(t+1, s)$-cover-free, we know that there exists at least one $i^{*}$ such that $\mathrm{id}_{i^{*}}^{*}=1$, but $\mathrm{id}_{i^{*}}=0$ for all vectors $\phi(\mathrm{ID})$ corresponding to the (at most $t$ ) extraction queries ID $\neq \mathrm{ID}^{*}$. Intuitively, such an index $i^{*}$ is hence chosen with probability at least $1 /\left|S^{*}\right|=1 / s \geq 1 / n$, and conditioned on this, the simulation 
is easily seen to be perfect. Formally, we let Win PKE $_{\text {and }}$ Win $_{\mathrm{IBE}}$ be the events that $\mathcal{B}$ and $\mathcal{A}$ guess the bit in the respective security games. Then,

$$
\begin{aligned}
& \mathrm{P}\left[\text { Win }_{\mathrm{PKE}}\right]=\mathrm{P}\left[\text { Win }_{\mathrm{PKE}} \wedge \text { bad }=\text { false }\right]+P\left[\text { Win }_{\mathrm{PKE}} \wedge \text { bad }=\text { true }\right] \\
& \geq \mathrm{P}[\mathrm{bad}=\mathrm{false}] \cdot \mathrm{P}\left[\text { Win }_{\mathrm{PKE}} \mid \text { bad }=\text { false }\right] \\
&+\mathrm{P}[\text { bad }=\text { true }] \cdot P\left[\text { Win }_{\mathrm{PKE}} \mid \text { bad }=\text { true }\right] .
\end{aligned}
$$

Now, clearly, $P[$ bad $=$ true $]=1-P[$ bad $=$ false $]$, and $P[$ Win $P$ PKE $\mid$ bad $=$ true $] \geq$ $\frac{1}{2}$, since $\mathcal{B}$ outputs a random bit if bad is true. Moreover, one can verify that $\mathrm{P}[\mathrm{bad}=\mathrm{false}] \geq \frac{1}{n}$, and, as the simulation is perfect, $\mathrm{P}\left[\mathrm{Win}_{\mathrm{PKE}} \mid \mathrm{bad}=\mathrm{false}\right]=$ $\mathrm{P}[\mathrm{Win}$ IBE]. Formalizing these last two argument actually requires some (standard) extra work, using the fact that all random coins are independent of the choice of $i^{*}$, but we dispense with the details in this version. Plugging in terms into the above concludes the proof.

\subsection{Full Security of GLW}

We note that the above proof strategy used in Theorem 2 fails when we do not know the challenge identity $\mathrm{ID}^{*}$ at the point in time when the reduction $\mathcal{B}$ sets the public parameters pp. However, an additional syntactic requirement on the underlying cryptosystem PKE yields full security, as we show below. This requirement is captured by the following definition.

Definition 3 (Weak Multi-Key Malleability). We say that PKE is weakly $n$-key malleable if there exists an efficient algorithm Simulate such that for all messages $m$, all $I \subseteq[n]$, and all $i \in I$, the probability distributions $D_{0}$ and $D_{1}$ are computationally indistinguishable, where with $(\mathbf{p k}, \mathbf{s k}) \stackrel{\$}{\leftarrow} \mathrm{Gen}^{n}, D_{b}$ consists of $\left(\mathbf{p k}, \mathbf{s k}[[n] \backslash\{i\}], c_{b}\right)$ such that

(1) $c_{0} \stackrel{\$}{\leftarrow} \operatorname{Enc}\left(\prod_{i \in I} \mathbf{p k}[i], m\right)$;

(2) $c \stackrel{\$}{\leftarrow} \operatorname{Enc}(\mathbf{p k}[i], m), c_{1} \stackrel{\$}{\leftarrow} \operatorname{Simulate}(i, I, c, \mathbf{p k}, \mathbf{s k}[[n] \backslash\{i\}])$.

In other words, given a ciphertext $c$ encrypting with public key $\mathbf{p k}[i]$ (where $i$ is part of some set $I$ ) an arbitrary unknown message $m$, we can efficiently generate a ciphertext $c^{\prime}$ encrypting the same message $m$ under the product of the keys $\mathbf{p k}[j]$ for $j \in I$ without knowing the secret key $\mathbf{s k}[i]$, but still possibly using $\mathbf{s k}[j]$ for $j \neq i$. The resulting ciphertext has the right distribution in the eyes of a computationally bounded distinguisher.

The proof of the following theorem follows a similar approach to the one of Theorem 2, and is deferred to the full version.

Theorem 3 (Full Security of GLW). Assume that PKE is ind-cpa-secure and weakly $n$-key malleable, and that $\phi$ is $(t+1, s)$-cover free. Then, the GLW construction is t-cpa-secure. 


\subsection{Instantiation from DDH}

We present a simple instantiation of the above paradigm based on the Decisional Diffie-Hellman (DDH) assumption and the ElGamal cryptosystem. The resulting scheme has smaller ciphertexts than earlier BC-IBE schemes [22]15], both requiring three group elements.

Concretely, let $G$ be a group with prime order $|G|=q$ and generator $g$. Recall that the ElGamal cryptosystem has secret key sk $\stackrel{\&}{\leftarrow} \mathbb{Z}_{q}$ and public key $\mathrm{pk}=g^{s k}$.

For a message $m \in G$, the encryption algorithm is $\operatorname{Enc}(\mathrm{pk}, m)=\left(g^{r}, m \cdot \mathrm{pk}^{r}\right)$, where $r \stackrel{\&}{\leftarrow} Z_{q}$, whereas $\operatorname{Dec}\left(\right.$ sk, $\left.\left(c_{1}, c_{2}\right)\right)=c_{2} \cdot c_{1}^{- \text {sk }}$. ElGamal is easily shown to be ind-cpa-secure under the DDH assumption. Moreover, we observe the following two properties of the ElGamal cryptosystem:

1. ElGamal admits a secret-key to public-key homomorphism $\mu: \mathbb{Z}_{q} \rightarrow G$ where $\mu(x)=g^{x}$, and $n$-correctness is satisfied for any $n$.

2. Moreover, it satisfies (perfect) weak $n$-key malleability: Namely, just consider the algorithm that for all $I \subseteq[n], i \in I$, and secret- and public-key vectors sk and pk, outputs

$$
c^{*}=\operatorname{Simulate}\left(i, I, \mathbf{p k}, \mathbf{s k}[[n] \backslash\{i\}],\left(c_{1}, c_{2}\right)\right)=\left(c_{1}, c_{2} \cdot c_{1}^{\sum_{j \neq i} \mathbf{s k}[j]}\right) .
$$

In particular, the resulting IBE scheme with identities $\mathcal{I D}$ obtained by plugging ElGamal into the GLW construction, for any $(t+1, s)$-cover-free map $\phi: \mathcal{I D} \rightarrow$ $\{0,1\}^{n}$, is as follows, and Theorem 3 implies its $t$-ibe-cpa security under the DDH assumption. (The decryption algorithm remains the same as in the original ElGamal scheme.)

\begin{tabular}{|c|c|c|}
\hline IBEGen: & IBEExtract $(\mathrm{msk}=\mathrm{sk}, \mathrm{ID})$ & IBEEnc $(\mathrm{pp}=(g, \mathbf{p k}), \mathrm{ID}, m)$ \\
\hline $\begin{array}{l}g \stackrel{\mathbb{S}}{\leftarrow} G \\
\mathbf{s k} \stackrel{\$}{\leftarrow} \mathbb{Z}_{q}^{n}, \mathbf{p k}[i] \leftarrow g^{\mathbf{s k}[i]} \\
\mathrm{pp} \leftarrow(g, \mathbf{p k}), \text { msk } \leftarrow \mathbf{s k} \\
\text { Return (pp, msk })\end{array}$ & $\begin{array}{l}{\left[\mathrm{id}_{1}, \ldots, \mathrm{id}_{n}\right] \leftarrow \phi(\mathrm{ID})} \\
\mathrm{sk}_{\mathrm{ID}} \leftarrow \sum_{i=1}^{n} \mathrm{id}_{i} \cdot \operatorname{sk}[i] \\
\operatorname{Return~sk}_{\mathrm{ID}}\end{array}$ & $\begin{array}{l}{\left[\operatorname{id}_{1}, \ldots, \mathrm{id}_{n}\right] \leftarrow \phi(\mathrm{ID})} \\
r \stackrel{\leftrightarrow}{\leftarrow} \mathbb{Z}_{q} \\
c \leftarrow\left(g^{r}, m \cdot \prod_{i=1}^{n} \mathbf{p k}[i]^{r \cdot \mathrm{id}_{i}}\right) \\
\text { Return } c\end{array}$ \\
\hline
\end{tabular}

\subsection{Instantiations from LWE and QR}

We achieve an additional instantiation of the above paradigm starting from the GPV cryptosystem [20. We thus achieve BC-IBE based on the learning with errors (LWE) assumption (with polynomial modulus for selective security and subexponential modulus for full semantic security).

Additionally, we achieve an instantiation under the quadratic residuosity (QR) assumption based on a simplified variant of the QR-based PKE scheme from [22]. We defer the details of both of these constructions to the full version. 


\section{Construction from Multi-key Malleability}

\subsection{Bounded-IBE Construction}

We present a further construction of BC-IBE from PKE schemes which satisfy a different notion of key malleability than the one given above, which we first introduce. Our notion requires that given an encryption of a message under one public key, we are asking for the ability to produce a new ciphertext of the same message which decrypts under a combination of secret keys (e.g., the product) for which we only know the corresponding public keys. Note that we are only asking for decryptability under the combination of the secret keys. In particular, in contrast to the above notion of weak key-malleability, the distribution of the resulting ciphertext may not be a valid encryption under some well-defined combination of the corresponding public keys, and moreover, we require ability to compute this ciphertext without knowledge of any secret keys.

Definition 4 (Multi-Key Malleability). Let PKE be a public-key encryption scheme. We say that PKE is $n$-key malleable if there exist algorithms Modify and Combine such that the following properties hold:

(i) For all valid messages $m$, all $I \subseteq[n]$, and all $i \in I$, the following probability is negligible (taken over the coins of Enc):

$$
\mathrm{P}\left[\begin{array}{l}
(\mathbf{p k}, \mathbf{s k}) \stackrel{\mathbb{\&}}{\leftarrow} \operatorname{Gen}^{n}, c \stackrel{\$}{\leftarrow} \operatorname{Enc}(\mathbf{p k}[i], m), \quad: \operatorname{Dec}\left(\operatorname{Combine}(I, \mathbf{s k}), c^{\prime}\right) \neq m \\
c^{\prime} \stackrel{\&}{\leftarrow} \operatorname{Modify}(i, I, \mathbf{p k}, c)
\end{array}\right] .
$$

(ii) For all $I \subseteq[n]$, Combine $(I, \mathbf{s k})$ does not depend on $\mathbf{s k}[i]$ for $i \notin I$.

(iii) For all $I \subseteq[n]$ and all valid public-key / secret-key vectors (pk, sk), for all $i, j \in I$, the values $\operatorname{Modify}(i, I, \mathbf{p k}, \operatorname{Enc}(\mathbf{p k}[i], m))$ and

$\operatorname{Modify}(j, I, \mathbf{p k}, \operatorname{Enc}(\mathbf{p k}[j], m))$ are equally distributed.

We note that Property (iii) above is not really necessary (a computational relaxation would suffice), but will make the presentation somewhat simpler and is true in the only instantiation we give below.

The IBE CONSTRUCtion AND ITS SECURITy. For an identity map $\phi: \mathcal{I D} \rightarrow$ $\{0,1\}^{n}$, we now propose a construction of an identity-based encryption scheme $\mathrm{IBE}=(\mathrm{IBEGen}, \mathrm{IBEExtract,}$ IBEEnc, IBEDec) from an $n$-key malleable encryption scheme PKE $=($ Gen, Enc, Dec). The decryption algorithm is unaltered, i.e., $\mathrm{IBEDec}=\mathrm{Dec}$, and moreover the construction consists of the following algorithms. (Note that the choice of $i$ as $\min \{\phi(\mathrm{ID})\}$ below within IBEEnc is purely arbitrary.)

\begin{tabular}{|c|c|c|}
\hline IBEGen: & IBEExtract $(\mathrm{msk}=\mathbf{s k}, \mathrm{ID})$ & IBEEnc $(\mathrm{pp}=\mathbf{p k}, \mathrm{ID}, m)$ : \\
\hline$(\mathbf{p k}, \mathbf{s k}) \stackrel{\$}{\leftarrow} \mathrm{Gen}^{n}$ & $\left.\overline{\text { sk ID }_{\text {I }} \leftarrow \text { Combine }(\phi(I D), \text { sk }}\right)$ & $\overline{i \leftarrow \min \{\phi(\text { ID })\}}$ \\
\hline $\begin{array}{l}\text { msk } \leftarrow \text { sk } \\
\text { pp } \leftarrow \text { pk } \\
\text { Return (msk, pp) }\end{array}$ & Return sk & $\begin{array}{l}c^{\prime} \stackrel{\$}{\leftarrow} \operatorname{Enc}(\mathbf{p k}[i], m) \\
c \leftarrow \operatorname{Modify}\left(i, \phi(\mathrm{ID}), \mathbf{p k}, c^{\prime}\right) \\
\operatorname{Return} c\end{array}$ \\
\hline
\end{tabular}


Correctness of the scheme follows by Property (i) above. The following theorem establishes security of our new construction. The proof is deferred to the full version.

Theorem 4. Assume that PKE is ind-cpa-secure and $n$-key malleable, and that $\phi$ is $(t+1, s)$-cover free. Then, IBE is t-ibe-cpa-secure.

\subsection{NTRU-Based Instantiation and Fully-Homomorphic IBE}

We provide an instantiation of the above constructing using the multi-key homomorphic properties of NTRU-based public-key encryption [28, which we first review. For some parameters $r, n$ and $q$ (where $q$ is a prime), consider the ring of polynomials $R=\mathbb{Z}[x] /\left(x^{r}+1\right)$, and let $\chi$ be a $B$-bounded distribution on $R$, i.e., with overwhelming probability, $\chi$ samples a polynomial from $R$ whose coefficients are all at most $B$ in absolute value. All operations on polynomials are to be understood as over the ring $R_{q}=R / q R$. The NTRU cryptosystem is such that key generation Gen samples $f, g \stackrel{\$}{\leftarrow} \chi$ subject to the constraint that $f \equiv 1(\bmod 2)$, and sets $\mathrm{pk}=2 g / f$ and $\mathrm{sk}=f$. (Possibly, $f$ needs to be resampled until it admits an inverse in $R_{q}$, and $\chi$ is such that this happens with good probability.) The message $b \in\{0,1\}$ is encrypted as

$$
\operatorname{Enc}(\mathrm{pk}, m)=h \cdot \mathrm{pk}+2 e+b,
$$

where $h, e \stackrel{\$}{\leftarrow} \chi$. Finally, decryption, given $c$, outputs Dec(sk, $c)=\mathrm{sk} \cdot c(\bmod 2)$. To see why decryption is correct, note that

$$
\mathrm{sk} \cdot c \equiv f \cdot(2 h \cdot g / f+2 e+b) \equiv 2 h \cdot g+2 e \cdot f+f \cdot b \quad(\bmod q) .
$$

If $B \leq \sqrt{q / 2} / r$, then all coefficients from $h \cdot g$ and $e \cdot f$ are of size at most $r^{2} B^{2}<q / 2$. Consequently, $2 h g$ and $2 e f$ only have even coefficients, and are 0 modulo 2 . And finally, $f \cdot b$ clearly always equals $b$ modulo 2 .

The scheme was proven ind-cpa-secure under a fairly ad-hoc assumption in 28, where it was also shown to have strong homomorphic properties we address below, and which we exploit for our construction.

The IBE Scheme. We turn now to building an IBE scheme from the above NTRU-based PKE scheme PKE using the above generic approach. In the following, we assume that $r$ is our security parameter, $q=2^{n^{\varepsilon}}$ for some constant $\varepsilon<1, B=\operatorname{poly}(r)$, and $n=\Theta\left(r^{\delta}\right)$ for some constant $\delta<1$.

We first show $\ell$-key malleability exploiting the multi-key homomorphic properties of NTRU shown in [28]. To this end, we define the algorithm Combine which given $I \subseteq[\ell]$ and $\mathbf{s k} \in R_{q}^{\ell}$ outputs

$$
\text { Combine }(I, \mathbf{s k})=\prod_{i \in I} \mathbf{s k}[i] .
$$

Moreover, we also define the (randomized) function Modify, which given $I \subseteq[\ell]$, $i \in I, c \in R_{q}$, and pk $\in R_{q}^{\ell}$, outputs

$$
\operatorname{Modify}(i, I, c, \mathbf{p k})=c+\sum_{j \in I \backslash\{i\}} h_{j} \cdot \mathbf{p k}[j],
$$


where $h_{j}$ for $j \in I \backslash\{i\}$ are sampled independently from the $B$-bounded distribution $\chi$ as above. Now, Properties (ii) and (iii) in Definition 4 are immediate to verify. Moreover, for Property (i), fix $I \subseteq[\ell]$ and $i \in I$, and pk, sk $\in R_{q}^{\ell}$, each consisting of $\ell B$-bounded polynomials as components, then define $c$ as

$$
c=\operatorname{Modify}(i, I, \operatorname{Enc}(\mathbf{p k}[i], b), \mathbf{p k})=\sum_{j \in I} h_{j} \cdot \mathbf{p k}[j]+2 e+b,
$$

and observe that

$$
\operatorname{Dec}(\text { Combine }(I, \mathbf{s k}), c)=\left(\prod_{i \in I} \mathbf{s k}[i]\right) \cdot\left(\sum_{j \in I} h_{j} \cdot \mathbf{p k}[j]+2 e+b\right) \quad(\bmod 2) .
$$

In particular,

$$
\begin{aligned}
\left(\prod_{i \in I} \mathbf{s k}[i]\right) & \left(\sum_{j \in I} h_{j} \cdot \mathbf{p k}[j]+2 e+b\right) \equiv \\
& \sum_{j \in I} 2 h_{j} \cdot g_{j} \cdot \prod_{i \in I \backslash\{j\}} f_{\ell}+\left(2 e \cdot \prod_{i \in I} f_{\ell}\right)+b \cdot\left(\prod_{i \in I} f_{\ell}\right) .
\end{aligned}
$$

Note that in the above sum, only products of at most $|I|+1 B$-bounded polynomials occurs. The coefficients of the resulting products have size at most $r^{|I|} \cdot B^{|I|+1}$, which (given previous parameter choices) is smaller than $q / 2$ as long as $|I|=o\left(n^{\varepsilon}\right)$. This yields correct decryption as no wraparound (modulo $q$ ) occurs.

\begin{tabular}{|c|c|c|}
\hline IBEGen: & IBEExtract $(\mathrm{msk}=\mathrm{sk}, \mathrm{ID}$ & $\sqrt{\text { JBEEnc }(p p=p k, ~ I D, ~} m):$ \\
\hline$\left\{\begin{array}{l}f_{1}, \ldots, f_{n} \stackrel{\$}{\leftarrow} \chi \\
\left(f_{i} \equiv 1(\bmod 2), f_{i} \in R_{q}^{*}\right) \\
g_{1}, \ldots, g_{n} \stackrel{\$}{\leftarrow} \chi \\
\text { msk } \leftarrow\left(f_{1}, \ldots, f_{n}\right) \\
\text { pp } \stackrel{\$}{\leftarrow}\left(2 g_{1} / f_{1}, \ldots 2 g_{n} / f_{n}\right) \\
\operatorname{Return}(\text { msk, pp })\end{array}\right.$ & $\begin{array}{l}\operatorname{sk}_{\mathrm{ID}} \leftarrow \prod_{i \in \phi(\mathrm{ID})} \mathbf{s k}[i] \\
\text { Return sk }\end{array}$ & $\left\{\begin{array}{l}h_{1}, \ldots, h_{n}, e \stackrel{\$}{\leftarrow} \chi \\
c \leftarrow \sum_{i \in \phi(\text { ID })} \mathbf{p k}[i] \cdot h_{i} \\
\quad+2 e+m\end{array}\right.$ \\
\hline
\end{tabular}

THE FINAL SCHEME. Overall, this yields to the following scheme, for any identity mapping $\phi: \mathcal{I D} \rightarrow\{0,1\}^{\ell}$ which is $(s, t+1)$-cover-free for some $s=o\left(n^{\varepsilon}\right)$, which is $t$-ind-cpa secure by Theorem 4 .

Fully-HomomorPHIC IBE. The above instantiation has additionally the property of being fully-homomorphic in the following sense:

Given encryptions IBEEnc(ID, $\left.m_{1}\right), \ldots$, IBEEnc(ID, $\left.m_{t}\right)$, and a function $f$ : $\{0,1\}^{t} \rightarrow\{0,1\}$, we can compute a ciphertext which decrypts to $f\left(m_{1}, \ldots, m_{t}\right)$ under sk ID using the homomorphic-evaluation procedures given in [28].

We note that in general one can provide a construction, along the lines given above, from multi-key fully-homomorphic encryption to fully-homomorphic identity-based encryption for bounded collusions. We defer a full discussion to the full version of this paper, noting in passing that the above is the only instantiation of this paradigm we are aware of. 


\section{Applications: Bounded CCA Security with Short Ciphertexts}

In this section, we revisit the generic transform by Boneh, Canetti, Halevi, and Katz [4] in the context of BC-IBE, and use it to obtain constructions of boundedCCA2 secure encryption schemes with short ciphertexts from any semantically secure scheme with a secret-key to public-key homomorphism.

THE BCHK TRANSFORM. Boneh et al 4] present a construction of an encryption scheme PKE = (Gen, Enc, Dec) from a selectively-secure IBE scheme IBE = (IBEGen, IBEExtract, IBEEnc, IBEDec) and a strong one-time signature scheme $S S=\left(\right.$ Gen $_{S S}$, Sign, Verify $)$. They then proceed to prove chosen-ciphertext security of the resulting PKE.

Of note is that in their reduction to the selective security of IBE, the reduction makes at most one IBEExtract query for each decryption query it receives from the adversary, and no other parameters change. Thus, their proof carries through exactly in the bounded-collusion case, yielding:

Theorem 5. If IBE is t-selective-ibe-cpa-secure, and if SS is strongly one-time secure, then PKE is $t$-CCA secure.

Applichtions. Using previous results, we directly obtain bounded CCA PKE constructions from DDH, QR, NTRU, and (standard) LWE using the constructions of the previous sections. In particular, note that only standard LWE is required as we only need selective security to instantiate the above paradigm. Moreover, the resulting DDH construction is essentially equivalent to the one presented in [10, and our construction thus provides an abstraction to obtain the same construction.

As an example, we give the $t$-CCA PKE based on the NTRU assumption that comes from applying Theorem 5 to the BC-IBE of Section 4.2. (Here the parameters $q, \chi, R_{q}^{*}$ are defined as in that section.)

\begin{tabular}{|c|c|c|}
\hline Gen: & & \\
\hline $\begin{array}{l}f_{1}, \ldots, f_{n} \stackrel{\$}{\leftarrow} \chi \\
\quad\left(f_{i} \equiv 1(\bmod 2), f_{i} \in R_{q}^{*}\right) \\
g_{1}, \ldots, g_{n} \stackrel{\$}{\leftarrow} \chi \\
\text { sk } \leftarrow\left(f_{1}, \ldots, f_{n}\right) \\
\text { pk } \leftarrow\left(2 g_{1} / f_{1}, \ldots, 2 g_{n} / f_{n}\right) \\
\text { Return }(\mathrm{pk}, \mathrm{sk}) .\end{array}$ & $\begin{array}{l}\left(\mathrm{skss}_{\mathrm{s}}, \mathrm{vk}_{\mathrm{ss}}\right) \stackrel{\$}{\leftarrow} \text { Gen }_{\mathrm{ss}} \\
h_{1}, \ldots, h_{n}, e \stackrel{\$}{\leftarrow} \chi \\
c \leftarrow \\
\sum_{i \in \phi\left(\mathrm{vkss}_{\mathrm{ss}}\right)} \mathbf{p k}[i] \cdot h_{i}+2 e+m \\
\sigma \stackrel{\$}{\leftarrow} \operatorname{Sign}\left(\mathrm{skss}_{\mathrm{ss}}, c\right) \\
\operatorname{Return}\left(\mathrm{vk}_{\mathrm{ss}}, c, \sigma\right)\end{array}$ & $\begin{array}{l}\text { If Verify }(\mathrm{vk}, c, \sigma)=0 \\
\text { then } \\
m \leftarrow \perp \\
\text { Else } \\
\text { sk }_{\mathrm{vk}} \leftarrow \prod_{i \in \phi(\mathrm{vk})} \mathbf{s k}[i] \\
m \leftarrow \mathrm{sk}_{\mathrm{vk}} \cdot c(\bmod 2)\end{array}$ \\
\hline
\end{tabular}

The ciphertext size of the CCA scheme generated by the BCHK transform is the same as the ciphertext size of the IBE scheme (and hence of the NTRU encryption scheme), plus a verification key and signature. Steinfeld et al. [31] show a (fully) CCA-secure construction based on NTRU; their ciphertext contains $k$ ciphertexts of the underlying NTRUEncrypt algorithm (where $k=\Theta(1)$ is a parameter that depends on the hardness assumption used, but is at least 4), and 
additionally a verification key, a signature, and a blinded message. (Since the NTRUEncrypt ciphertexts are polynomials in $R_{q}$, they will typically be much larger than the other values.) Thus, we obtain a constant-factor improvement in ciphertext size by moving to the bounded-query model, in addition to the conceptual simplicity of the proof.

Acknowledgments. The authors wish to thank Shafi Goldwasser for insightful feedback and motivating us to write the present paper.

The research of this paper was partially supported by NSF Contract CCF1018064. Moreover, this material is based on research sponsored by DARPA under agreement numbers FA8750-11-C-0096 and FA8750-11-2-0225. The U.S. Government is authorized to reproduce and distribute reprints for Governmental purposes notwithstanding any copyright notation thereon. The views and conclusions contained herein are those of the authors and should not be interpreted as necessarily representing the official policies or endorsements, either expressed or implied, of DARPA or the U.S. Government.

\section{References}

1. Agrawal, S., Boneh, D., Boyen, X.: Lattice basis delegation in fixed dimension and shorter-ciphertext hierarchical IBE. In: Rabin, T. (ed.) CRYPTO 2010. LNCS, vol. 6223, pp. 98-115. Springer, Heidelberg (2010)

2. Bellare, M., Desai, A., Pointcheval, D., Rogaway, P.: Relations among notions of security for public-key encryption schemes. In: Krawczyk, H. (ed.) CRYPTO 1998. LNCS, vol. 1462, pp. 26-45. Springer, Heidelberg (1998)

3. Boneh, D., Boyen, X.: Secure identity based encryption without random oracles. In: Franklin, M. (ed.) CRYPTO 2004. LNCS, vol. 3152, pp. 443-459. Springer, Heidelberg (2004)

4. Boneh, D., Canetti, R., Halevi, S., Katz, J.: Chosen-ciphertext security from identity-based encryption. SIAM Journal on Computing 36(5), 1301-1328 (2007)

5. Boneh, D., Franklin, M.: Identity-based encryption from the weil pairing. In: Kilian, J. (ed.) CRYPTO 2001. LNCS, vol. 2139, pp. 213-229. Springer, Heidelberg (2001)

6. Boneh, D., Gentry, C., Hamburg, M.: Space-efficient identity based encryption without pairings. In: 48th Annual Symposium on Foundations of Computer Science, pp. 647-657. IEEE Computer Society Press (October 2007)

7. Canetti, R., Halevi, S., Katz, J.: A forward-secure public-key encryption scheme. In: Biham, E. (ed.) EUROCRYPT 2003. LNCS, vol. 2656, pp. 255-271. Springer, Heidelberg (2003)

8. Cash, D., Hofheinz, D., Kiltz, E., Peikert, C.: Bonsai trees, or how to delegate a lattice basis. In: Gilbert, H. (ed.) EUROCRYPT 2010. LNCS, vol. 6110, pp. 523-552. Springer, Heidelberg (2010)

9. Cocks, C.: An identity based encryption scheme based on quadratic residues. In: Honary, B. (ed.) Cryptography and Coding 2001. LNCS, vol. 2260, pp. 360-363. Springer, Heidelberg (2001)

10. Cramer, R., Hanaoka, G., Hofheinz, D., Imai, H., Kiltz, E., Pass, R., Shelat, A., Vaikuntanathan, V.: Bounded CCA2-secure encryption. In: Kurosawa, K. (ed.) ASIACRYPT 2007. LNCS, vol. 4833, pp. 502-518. Springer, Heidelberg (2007) 
11. Cramer, R., Shoup, V.: Universal hash proofs and a paradigm for adaptive chosen ciphertext secure public-key encryption. In: Knudsen, L.R. (ed.) EUROCRYPT 2002. LNCS, vol. 2332, pp. 45-64. Springer, Heidelberg (2002)

12. Diffie, W., Hellman, M.E.: New directions in cryptography. IEEE Transactions on Information Theory 22(6), 644-654 (1976)

13. Dodis, Y., Fazio, N.: Public key broadcast encryption for stateless receivers. In: Feigenbaum, J. (ed.) DRM 2002. LNCS, vol. 2696, pp. 61-80. Springer, Heidelberg (2003)

14. Dodis, Y., Haitner, I., Tentes, A.: On the instantiability of hash-and-sign RSA signatures. In: Cramer, R. (ed.) TCC 2012. LNCS, vol. 7194, pp. 112-132. Springer, Heidelberg (2012)

15. Dodis, Y., Katz, J., Xu, S., Yung, M.: Key-insulated public key cryptosystems. In: Knudsen, L.R. (ed.) EUROCRYPT 2002. LNCS, vol. 2332, pp. 65-82. Springer, Heidelberg (2002)

16. Erdös, P., Frankel, P., Furedi, Z.: Families of finite sets in which no set is covered by the union of $r$ others. Israeli Journal of Mathematics 51, 79-89 (1985)

17. Fiat, A., Naor, M.: Broadcast encryption. In: Stinson, D.R. (ed.) CRYPTO 1993. LNCS, vol. 773, pp. 480-491. Springer, Heidelberg (1994)

18. Gafni, E., Staddon, J., Yin, Y.L.: Efficient methods for integrating traceability and broadcast encryption. In: Wiener, M. (ed.) CRYPTO 1999. LNCS, vol. 1666, pp. 372-387. Springer, Heidelberg (1999)

19. Garay, J.A., Staddon, J., Wool, A.: Long-lived broadcast encryption. In: Bellare, M. (ed.) CRYPTO 2000. LNCS, vol. 1880, pp. 333-352. Springer, Heidelberg (2000)

20. Gentry, C., Peikert, C., Vaikuntanathan, V.: Trapdoors for hard lattices and new cryptographic constructions. In: Ladner, R.E., Dwork, C. (eds.) 40th ACM STOC Annual ACM Symposium on Theory of Computing, pp. 197-206. ACM Press, New York (May 2008)

21. Gentry, C., Sahai, A., Waters, B.: Homomorphic encryption from learning with errors: Conceptually-simpler, asymptotically-faster, attribute-based. Cryptology ePrint Archive, Report 2013/340 (2013), http://eprint.iacr.org/

22. Goldwasser, S., Lewko, A., Wilson, D.A.: Bounded-collusion IBE from key homomorphism. In: Cramer, R. (ed.) TCC 2012. LNCS, vol. 7194, pp. 564-581. Springer, Heidelberg (2012)

23. Goldwasser, S., Micali, S.: Probabilistic encryption. Journal of Computer and System Sciences 28(2), 270-299 (1984)

24. Gorbunov, S., Vaikuntanathan, V., Wee, H.: Functional encryption with bounded collusions via multi-party computation. In: Safavi-Naini, R., Canetti, R. (eds.) CRYPTO 2012. LNCS, vol. 7417, pp. 162-179. Springer, Heidelberg (2012)

25. Halevy, D., Shamir, A.: The LSD broadcast encryption scheme. In: Yung, M. (ed.) CRYPTO 2002. LNCS, vol. 2442, pp. 47-60. Springer, Heidelberg (2002)

26. Hoffstein, J., Pipher, J., Silverman, J.H.: NTRU: A ring-based public key cryptosystem. In: Buhler, J.P. (ed.) ANTS 1998. LNCS, vol. 1423, pp. 267-288. Springer, Heidelberg (1998)

27. Kumar, R., Rajagopalan, S., Sahai, A.: Coding constructions for blacklisting problems without computational assumptions. In: Wiener, M. (ed.) CRYPTO 1999. LNCS, vol. 1666, pp. 609-623. Springer, Heidelberg (1999)

28. López-Alt, A., Tromer, E., Vaikuntanathan, V.: On-the-fly multiparty computation on the cloud via multikey fully homomorphic encryption. In: Karloff, H.J., Pitassi, T. (eds.) 44th ACM STOC Annual ACM Symposium on Theory of Computing, pp. 1219-1234. ACM Press (May 2012) 
29. Naor, D., Naor, M., Lotspiech, J.: Revocation and tracing schemes for stateless receivers. In: Kilian, J. (ed.) CRYPTO 2001. LNCS, vol. 2139, pp. 41-62. Springer, Heidelberg (2001)

30. Shamir, A.: Identity-based cryptosystems and signature schemes. In: Blakely, G.R., Chaum, D. (eds.) CRYPTO 1984. LNCS, vol. 196, pp. 47-53. Springer, Heidelberg (1985)

31. Steinfeld, R., Ling, S., Pieprzyk, J., Tartary, C., Wang, H.: NTRUCCA: How to strengthen NTRUEncrypt to chosen-ciphertext security in the standard model. In: Fischlin, M., Buchmann, J., Manulis, M. (eds.) PKC 2012. LNCS, vol. 7293, pp. 353-371. Springer, Heidelberg (2012)

32. Waters, B.: Efficient identity-based encryption without random oracles. In: Cramer, R. (ed.) EUROCRYPT 2005. LNCS, vol. 3494, pp. 114-127. Springer, Heidelberg (2005) 